4 Mariela Bernabe-García ${ }^{a^{*}}, R D, P h D ;$ Raúl Villegas-Silva ${ }^{b}$, MD; Astrid Villavicencio-

5 Torres $^{\mathrm{c}}$, MD; Philip C. Calder ${ }^{\mathrm{d}}$, PhD; Maricela Rodríguez-Cruz ${ }^{\mathrm{a}}, \mathrm{PhD}$; Jorge Maldonado-

6 Hernández $^{\mathrm{a}}, \mathrm{PhD}$; Denisse Macías-Loaiza ${ }^{\mathrm{a}}, \mathrm{D}$.; Mardia López-Alarcón ${ }^{\mathrm{a}}, \mathrm{MD}, \mathrm{PhD}$; Patricia

7 Inda-Icaza ${ }^{\mathrm{e}}, \mathrm{RD}, \mathrm{PhD}$; Leonardo Cruz-Reynoso ${ }^{\mathrm{f}}, \mathrm{MD}, \mathrm{MSc}$.

\section{Enteral Docosahexaenoic Acid and Retinopathy of Prematurity: A Randomized}

\section{Clinical Trial}

9

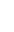

\title{
Affiliations:
}

${ }^{a}$ Unidad de Investigación Médica en Nutrición, Hospital de Pediatría, C.M.N. Siglo XXI, Instituto Mexicano del Seguro Social (IMSS), Ciudad de México, México.

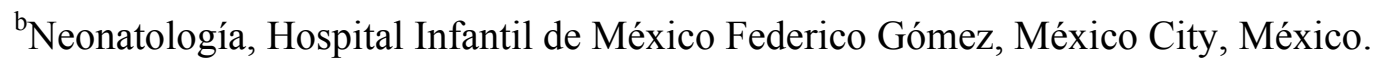

`Oftalmología Pediátrica, General Hospital, C.M.N. La Raza, IMSS, Ciudad de México, México.

${ }^{\mathrm{d} H u m a n}$ Development and Health Academic Unit, Faculty of Medicine, University of Southampton, Southampton General Hospital, Southampton, UK \& NIHR Southampton Biomedical Research Centre, University Hospital Southampton NHS Foundation Trust and University of Southampton, Southampton, UK.

eNutrición. Universidad Anáhuac México, Campus Norte, Huixquilucan, Estado de México, México

${ }^{\mathrm{f}}$ División de Pediatría, Hospital de Gineco-Obstetricia No.3, C.M.N. La Raza, IMSS, Ciudad de México, México. 
26 *Corresponding author: Mariela Bernabe-Garcia, Ph.D.

27 Unidad de Investigación Médica en Nutrición, Hospital de Pediatría, Centro Médico

28 Nacional Siglo XXI, IMSS. Av. Cuauhtémoc 330, Col. Doctores, Del. Cuauhtémoc,

29 México City, C. P. 06725 México.

30 Telephone:+52(55)56276900 Exts. 22483 \& 22484. Fax:+52(55)56276944.

31 E-mail:marielabernabe1@gmail.com

32

33

34

35

36

37

Clinical Trial registration: Effect of Enteral Administration of Docosahexaenoic Acid on Development of the Retinopathy of Prematurity. ID: NCT02683317.

https://clinicaltrials.gov/ct2/show/NCT02683317 


\section{Abstract}

Background and Aim: Retinopathy of prematurity (ROP) is a disorder of the retina of lowbirth-weight preterm infants that potentially leads to blindness. Docosahexaenoic acid (DHA), a component of fish oil, is protective in experimental models. Administration of fish oil as part of parenteral nutrition has shown inconsistent results. We aimed to test the effect of enteral DHA to prevent ROP development and/or severity, and to reduce hospital stay.

Methods: This was a double-blind, parallel clinical-trial. Preterm infants $(\mathrm{n}=110 ; 55$ per group) with birth weight $<1500 \mathrm{~g}$ but $\geq 1000 \mathrm{~g}$ were recruited in a level $3 \mathrm{NICU}$. Infants were randomized to receive $75 \mathrm{mg}$ of DHA/kg/day (DHA-group) or high oleic sunflower oil (control-group) for 14 days by enteral feeding. The effect of DHA was evaluated on any stage of ROP, severe ROP (stage $\geq 3$ ) incidence, and hospital stay. Groups were compared with relative risk and 95\% CI, Fisher's Exact, Student's t or Mann-Whitney U tests, as appropriate. Logistic regression was applied to adjust for confounders.

Results: There was no difference between the DHA and control groups in ROP risk (RR for $\mathrm{DHA}=0.79 ; 95 \% \mathrm{CI}, 0.49-1.27, P=0.33$ ). However, patients who received DHA showed lower risk for stage $3 \mathrm{ROP}$ than controls (RR for $\mathrm{DHA}=0.66$; $95 \% \mathrm{CI}, 0.44-0.99, P=0.03$ ). After adjusting for confounders, enteral DHA decreased the risk of stage $3 \mathrm{ROP}(\mathrm{OR}$ adj. $=$ $0.10 ; 95 \% \mathrm{CI}, 0.011-0.886 ; P=0.04)$. Hospital stay was not different between groups. Conclusion: Enteral DHA may reduce the incidence of stage 3 ROP.

Key words: ROP; DHA; omega-3 fatty acids; neonate; premature infant, retinopathy, preterm. 
Clincial Relevancy Statement

Severe retinopathy of prematurity (ROP) is a leading cause of visual impairment and childhood blindness. Results from animals suggest DHA supplementation may have a protective effect, although evidence from preterm infants given parenteral nutrition that contains DHA is inconclusive. Enteral DHA supplementation from the start of the enteral feeding may be a feasible strategy to prevent severe ROP.

\section{Introduction}

Retinopathy of prematurity (ROP) is a highly prevalent disorder of the developing retina of low birth weight preterm infants that potentially leads to blindness. ${ }^{1}$ Currently, it is among the leading preventable causes of childhood blindness. ${ }^{2,3}$ The development of the retinal vasculature occurs almost entirely in utero in a hypoxic environment. When the metabolic demands of the developing retina exceed the oxygen supplied by the choroidal circulation, angiogenesis is stimulated through vasoactive factors such as insulin-like growth factor (IGF)-1, erythropoietin and vascular endothelial growth factor (VEGF), in addition to maternally derived factors. ${ }^{4}$

At birth, the loss of placental and maternal growth factors plus an increase of oxygen supply, suppress retinal growth factors, leading to cessation and retraction of the development of retinal vessels, or vaso-obliteration. ${ }^{5}$ As the preterm infant grows, the avascular retina undergoes neovascularisation due a subsequent up-regulation of cyclooxygenase-2 (COX-2), IGF-1 and VEGF, resulting in abnormal vascular overgrowth into the vitreous, retinal haemorrhages and folds, dilated and tortuous posterior vessels, up to retinal detachment. ${ }^{6}$ 
Strategies for prevention of ROP include timely detection and avoiding high fluctuations in tissue oxygen saturation $\left(\mathrm{SpO}_{2}\right){ }^{7}$ However, this approach is not fully effective, probably because ROP is a multi-factorial disease. Treatments include laser or cryotherapy, ${ }^{8}$ but these are destructive and they do not increase good vision $(>20 / 40)$, and medications such as anti-VEGF are still under evaluation. ${ }^{9,10}$ Therefore, further safer approaches for preventing and treating ROP are required.

It is well established that the omega-3 long-chain polyunsaturated fatty acids (LC-PUFA) docosahexaenoic acid (DHA) and eicosapentaenoic acid (EPA) play a key role in the protection against inflammation and oxidative stress. ${ }^{11,12}$ DHA and EPA are found in oily fish, many algae and fish oil. Inclusion of DHA and EPA in the diet of mice with oxygeninduced retinopathy decreased inflammation, neovascularisation and obliteration of retinal vessels. ${ }^{13}$ However, studies investigating the effect of DHA and EPA provided in fish oil administered as part of parenteral nutrition $(\mathrm{PN})$ to human preterm infants have produced inconsistent findings, either demonstrating prevention of ROP or no effect. ${ }^{14,15}$ To our knowledge there are no published studies of enteral administration of omega-3 LC-PUFA to prevent ROP in human infants. The hypothesis of this study was that enteral DHA will lead to a reduction in the incidence of ROP, severe ROP in preterm infants, and hospital stay.

\section{Materials and methods}

A randomized double-blind parallel group trial was conducted in a Hospital of GynaecoObstetrics, IMSS in Mexico City. Eligibility criteria were preterm neonates, with birth weight $<1500 \mathrm{~g}$ but $\geq 1000 \mathrm{~g}$, and a functional gastrointestinal tract. Those neonates with congenital malformations, need for major surgery, intraventricular haemorrhage grade >II 
to preclude the potential risk of increasing its severity, or whose mother was taking omega3 supplements were excluded. Although DHA does not increase bleeding risk, ${ }^{16}$ the intervention was suspended due to persisting bleeding at any level, platelet count $<80,000$ $\mathrm{mm}^{3}$, or its decrease by $50 \%$ from 3 prior days, or critical illness. This work was carried out in accordance with The Code of Ethics, ${ }^{17}$ and it was approved by the National Research and Ethics Committee (IMSS N²015-785-051). Registration on Clinical Trials.gov was done prior to the enrolment of the first patient (ID NCT02683317). Written informed consent was obtained from both parents along with the signature of 2 witnesses, prior to inclusion of each infant.

Preterm infants received 14 days of either a daily dose of $75 \mathrm{mg}$ of DHA/kg of baseline body weight from a DHA-rich algal oil, diluted in high oleic sunflower oil as vehicle (life'sDHA ${ }^{\circledR}$, DSM Nutritional Products Inc., Parsippany, NJ, USA) or sham oil (high oleic sunflower oil prepared by PROGELA SA (Mexico City, Mexico). The DHA dose was estimated to approximately mimic a high but still physiological content of DHA at $1 \%$ weight percentage of total fatty acids $(\% \mathrm{wt} / \mathrm{total} \mathrm{wt})$ in human milk. ${ }^{16}$ Sham and DHA oils were similar in volume (maximum $215 \mu \mathrm{L} /$ dose), colour and consistency to blind all staff. The fatty acid composition of two oils was measured by gas chromatography (Table 1). Each dose was administered as a bolus through an orogastric tube, before human milk or formula by the attending nurse, starting with the first enteral feeding after birth, referred to as baseline. All doses were prepared with aseptic technique by research staff who did not participate in the fieldwork. Random Allocation Software was used with block sizes of 10 patients, on a 1:1 ratio. ${ }^{18} \mathrm{~A}$ code was assigned to each intervention, stored and sequentially numbered in opaque envelopes by researcher staff who prepared doses. The treatment was assigned after parents gave their written informed consent. 
Incidence of ROP was defined as any stage of ROP identified in one or both eyes. Medical screening was carried out by a pediatric ophthalmologist, blinded to our intervention, using the zone I to III borders and clock hours to describe the location and extent of ROP, the stage (1 to 5), and the presence of plus disease. The latter was defined as dilation and tortuosity of the posterior retinal blood vessels. All patients received comfort measures such as analgesic pre-treatment, lubrication of the cornea, and pacifiers. ${ }^{19,20}$ Severity of ROP was defined as any stage with plus, or stages 3 to 5 with or without plus disease, which have a higher risk for vision impairment, including blindness. This was identified between the first evaluation carried out at 4-5 weeks after birth and throughout hospital stay. After discharge, follow-up continued until remission of ROP or 45 weeks of corrected gestational age. ${ }^{19-21}$ Laser treatment was indicated for those infants with any stage of ROP with plus disease, or stage 3 with/without plus disease; aggressive posterior disease; threshold ROP and prethreshold ROP according to the ETROP guidelines. ${ }^{20,22,23}$ The proportion of infants with treated ROP was also registered. Duration of hospital stay was considered from birth until discharge in days.

Bleeding events such as intraventricular haemorrhage grade $\geq$ II, gastric bleeding, vomiting events during the intervention period, and mortality were recorded as potential adverse events.

Clinical variables, feeding and medication were recorded as risk factors. Severity of disease was scored with the clinical risk index for babies (CRIB). ${ }^{24}$ The $\mathrm{SpO}_{2}<85 \%$ and $\mathrm{SpO}_{2}>95 \%$ were the alarm limits, recorded from the delivery room until supplementary oxygen was no longer required. The routine management followed the neonatal resuscitation program, with goal limits of oxygen saturation between $88 \%$ to $93 \%{ }^{25}$ 
The fatty acid profile in erythrocytes is considered a biomarker of the composition of peripheral tissues and retina. ${ }^{26}$ Thus, a venous blood sample was collected into EDTA tubes. To avoid additional punctures due to this study, consultants were asked to schedule the blood collection of the sample when it was ordered for clinical tests, before the first enteral feeding. Blood was not sampled if infants received a previous transfusion, as this is a modifier factor of the fatty acid profile. ${ }^{27}$ Blood was processed and analyzed as reported elsewhere. ${ }^{28,29}$ Briefly, fatty acids were measured by gas chromatography (7820A, Agilent Technologies, Santa Clara, CA, USA) using a standard for each fatty acid and heptadecanoic acid as internal standard. ${ }^{30}$ The fatty acid profile was also measured in human milk obtained every week. Results are presented as \%wt/total wt. Fatty acids contained in formula and parenteral nutrition were estimated from manufacturer's information.

\section{Statistical Analyses}

Data distribution was determined with Kolmogorov-Smirnov test. To identify differences between groups, relative risk (RR) with 95\% confidence interval $(95 \% \mathrm{CI})$, Fisher`s Exact and Chi-square, Student's-t or Mann-Whitney-U tests were used as appropriate.

Multivariate logistic regression was performed to adjust for confounders. SPSS software v.24 was used to statistical analysis. $P$ value $<0.05,2$-sided was considered as significant. Intention to treat analysis performed if infants received at least one dose of either intervention.

Sample size for ROP incidence was estimated with a 2-proportions formula according to a previous report, where $\mathrm{P} 1=28 \%$ and $\mathrm{P} 2=5 \%,{ }^{31} 2$-sided $\alpha=0.5$, sample power of $80 \%$ and $27 \%$ of drop-outs, as was seen in our previous study. A sample size of 55 patients per group was obtained. 


\section{Results}

182

183

184

185

186

Patients were recruited from February 2016 to October 2017 when the sample size was reached. Follow-up finished in January 2018. One hundred and ten preterm infants finished the study from 143 randomized infants (Fig. 1). The number of dropouts was not different between the DHA and control groups ( $26 \%$ vs. $20 \%, P=0.55)$. Preterm infants from the DHA group had higher birth weight than those from the control group, but weight did not differ between groups at baseline. Likewise, oleic acid in erythrocytes was higher but arachidonic acid and EPA were lower in the DHA group (Table 2); other characteristics at birth and baseline were similar between groups.

There was no difference on the incidence of any stage of ROP between patients in the DHA and control groups (Any ROP $19 / 55$ vs. $24 / 55, P=0.43)$, nor in the RR for ROP $(R R=0.79$; 95\% CI, $0.49-1.27, P=0.33$ ). However, among those infants who developed any stage of ROP, the incidence and risk for stage 3 ROP were lower in infants in the DHA group than the control group: $11 / 19$ vs. $21 / 24(P=0.04)$ and $\mathrm{RR}=0.66 ; 95 \% \mathrm{CI}, 0.44-0.99(P=0.03)$ respectively. This means a relative effect size of $34 \%$. This protective effect remained significant after adjusting for confounders (Table 3 ). There were no cases of ROP stage 4 or 5 corresponding to partially detached or detached retina. There was no difference in the proportion of infants who received treatment for ROP between the DHA and control groups (7/55 vs. $9 / 55, P=0.79)$.

Intention to treat analysis was carried out on 70 patients from the DHA group and 66 patients from the control group (Fig. 1). There were no differences between groups for the incidence and risk of all stages of ROP (34/70 vs. $35 / 66, P=0.61$ and $\mathrm{RR}=0.92 ; 95 \% \mathrm{CI}$, $0.66-1.28, P=0.61$ respectively), or for stage $3 \mathrm{ROP}(26 / 34$ vs. $32 / 35, P=0.11 ; \mathrm{RR}=0.84$; 
$95 \%$ CI, $0.68-1.03, P=0.09$ respectively). The median duration of hospital stay was not different between DHA and control groups (52 days [26-95] compared with 52 days [27107], $P=0.45$, respectively).

There was no difference between DHA and control groups with regard to the incidence or risk to develop intraventricular haemorrhage grade $\geq \mathrm{II}(30 / 55$ vs. $37 / 55, P=0.24$ and $\mathrm{RR}=$ $0.81 ; 95 \% \mathrm{CI}, 0.60-1.10, P=0.17$ ) or gastric bleeding $8 / 55$ vs. $13 / 55, P=0.33$ and $\mathrm{RR}=$ $0.62,95 \%$ CI, $0.28-1.37, P=0.23$; respectively. Similarly, the incidence of patients who presented vomit during the intervention was not different between DHA and control groups ( $1 / 55$ vs. $7 / 55, P=0.21)$. Total mortality during hospitalization was $4.9 \%$, with no difference between DHA and control groups (3/70 vs. 4/66 infants, $P=1.00)$.

The number of events of apnea was lower in the DHA group compared with the control group (Table 4). All infants received enteral formula complementing human milk or only formula during the study, and 70\% of infants received a lipid emulsion by PN without DHA nor EPA, but only $11 \%$ received human milk with no differences between groups (Table 4). Additional enteral DHA from mentioned sources was calculated for each patient, and then compared between groups, but there were no differences in those nor in fatty acid profile from enteral or parenteral feed (data not shown).

\section{Discussion}

The findings of this study demonstrate that enteral DHA prevents stage 3 ROP in preterm infants after adjusting for known confounders. Since stage 3 ROP can progress to a traccional retinal detachment, which can result in functional of complete blindness, this feasible strategy may be clinically useful. ${ }^{1}$ We previously demonstrated the pharmacological effect of enteral DHA to modulate inflammation and improve clinical 
outcomes in critically ill neonates, ${ }^{28,29,32,33}$ but to our knowledge, there are no published studies that evaluate the efficacy of enteral DHA on the development or severity of ROP, when given as a single intervention.

In the retina, arachidonic acid (AA) is a substrate of COX-2 increasing the production of prostaglandin $(\mathrm{PG}) \mathrm{E}_{2}$ and thromboxane $(\mathrm{TX}) \mathrm{A}_{2}$, resulting in the formation of pathological retinal neovessels, and microvascular degeneration, ischemia and oxidant stress, with death of retinal endothelial cells, respectively ${ }^{34-38}$ As the levels of antioxidants are low in the retina of preterm infants, oxygen-mediated lipid peroxidation increases the levels of isoprostanes and nitric oxide, resulting in impaired retinal circulation and vascular integrity, and then in vaso-proliferation. ${ }^{34}$ Conversely, when omega-3 LC-PUFA substrate is available, the retina can be protected through the biosynthesis of DHA-derived resolvins and protectins with potent anti-inflammatory and inflammation resolving effects. ${ }^{34,39}$ Moreover, the 5-LOX oxidation of DHA to 4-hydroxy-DHA, directly inhibits endothelial cell proliferation and sprouting angiogenesis via PPAR-gamma, independent of any antiinflammatory effect. ${ }^{40}$ Omega-3 LC-PUFA also exert anti-angiogenic effects through $\mathrm{PGE}_{3}$ from EPA with lower biological potency than $\mathrm{PGE}_{2}{ }^{41}$. Mice fed with $2 \%$ of omega-3 LCPUFA (including both DHA and EPA) had decreased production of TNF-alpha, resulting in decreased neovascularisation and less obliteration in retinal vessels. ${ }^{13}$ DHA was also able to decrease isoprostanes in a neonatal experimental model. ${ }^{42}$

In human preterm infants there are a small number of randomized clinical trials that have administered fish oil (a source of EPA and DHA), as part of PN for approximately two weeks of intervention with inconclusive results on ROP. One study reported less frequency of ROP requiring laser therapy, ${ }^{43}$ while a second study observed lower incidence of ROP at any stage, but no difference in the need for laser photocoagulation as treatment. ${ }^{31}$ The other 
252 studies found no difference in the development of ROP compared to control groups. ${ }^{44-46}$ It

253 is probable that the low omega 3 intake by our population along with the lack of breastmilk

254 intake, can lead to tissue DHA depletion of our patients at baseline and the increase in

255

256

257

258 exogenous DHA supply through our intervention lead to the positive findings with enteral DHA. Several infants with $\geq 3$ stage without plus in both groups presented spontaneous remission and did not receive treatment. In the current study, the duration of hospital stay was not different between groups, consistent with other reports. ${ }^{12,31}$

Although birth weight was statistically higher in infants in the DHA group, at study baseline the difference was smaller and no longer statistically or clinically significant. ROP has been associated to elevated oxygen saturation and extreme changes between hypoxia and hyperoxia, and since apnea events are a cause of hypoxia treated with hyperoxia, the higher number of apnea events in the control group was considered a major confounder. ${ }^{47}$ However, a recent study did not find a greater number of apnea events among infants with severe ROP, after accounting for gestational age. Authors mentioned it is likely that retinal vascular pathology is related to both hyperoxia/hypoxia and the phase of development at which aberrant $\mathrm{SpO}_{2}$ occurs. ${ }^{48}$ Nonetheless, apnea was also included into the multivariate analysis. Human milk is a known to be a protective factor for ROP development, ${ }^{15}$ but in the current study there was low availability of human milk because mothers found it difficult to breastfed, were critically ill or received contraindicated medication for breastfeeding.

AA and EPA were lower in erythrocytes from infants in the DHA group at baseline. It is generally accepted that AA is a substrate for inflammatory mediators, while EPA has antiinflammatory properties. ${ }^{35}$ However, recently it was reported that lower AA levels in plasma phospholipids were associated to ROP development, ${ }^{49}$ which is contrary to our 
hypothesis. Thus, the possible effect of AA on ROP remains elusive and the difference in content of AA at baseline is a limitation. However, erythrocyte AA and EPA at baseline were also taken into account in the multivariate analysis and the effect of DHA on stage 3 ROP remained significant. Erythrocyte oleic acid was higher in the DHA group at baseline, but its effect on stage 3 ROP was not significant in the multivariate model, and therefore it was not considered as a confounder.

Although a sample size calculation was done a priori, the incidence of ROP was higher in our study than for the data used to estimate power, which is a limitation. However, our power for stage 3 ROP remained high (96\%). Another limitation of our study is the timing of the start of the intervention, which was defined by the start of enteral feeding. It is possible that the efficacy of DHA would have been greater if the intervention started during the first hours after birth, similar to the administration of fish oil by PN in the previously mentioned studies. However, the start and suspension of enteral feeding were left to the discretion of attending neonatologists, considering the high risk to develop enteral intolerance and necrotizing enterocolitis in preterm infants, which is also reflected in the study dropouts. Although preterm infants with birth weight $<1000 \mathrm{~g}$ have the highest risk of ROP, they also are prone to gastrointestinal complications, thus our results are not generalizable to younger infants weighing less than $1000 \mathrm{~g}$.

Despite its limitations, this study provides evidence that enteral DHA administration used at a high dose found in human milk can be effective to prevent stage $3 \mathrm{ROP}$, and consequently the risk of severe ROP. PN usually is used in premature infants for nutritional support, but it also has been associated with liver disease and bloodstream infections. ${ }^{50}$ Thus, enteral nutriton should be established as soon as possible. These results show that enteral DHA is useful for preterm infants who start their supplementation as soon as they 
receive enteral feeding. A strength of the current study is that the dose of DHA was adjusted per kilogram of body weight, which gives comparability between subjects in the DHA group. This dose does not cause severe adverse effects, according to these results and to previous studies. ${ }^{28,29}$

Preventing and treating childhood blindness is a priority since it results in altered psychosocial/neurobehavioral development, economic dependence and low quality of life related to incapacity to work. Furthermore, the primary carer is also significantly affected, increasing the economic burden on family, society, and health care systems. ${ }^{8}$ Thus, ROP is highly disabling with no effective treatments. New prevention and treatment options need to be identified. Omega-3 LC-PUFA delivered by PN has been shown to have inconsistent findings, and the current study suggests that enteral DHA may offer such an option. In conclusion, the DHA delivered by enteral feeding may be an approach to prevent stage 3 ROP in preterm infants.

\section{Acknowledgements}

We thank the parents who agreed to participate on behalf of their newborn. We also thank Dr Juan Bravo-Ortiz, Dr Jose Magdaleno-Lara, Dr Paola Dominguez-Vallejo, neonatologists and nursery staff for their contribution and invaluable support.

Conflict of Interest Statement: The authors have no conflicts of interest relevant to this article to disclose. This work was supported by research grants Salud-2015-2-261765 from the Consejo Nacional de Ciencia y Tecnología, CONACYT, and FIS/IMSS/PROT/G15/1462 from IMSS (both to Dr MBG). Funders had no additional role in the performance, analysis, preparation, or approval of the manuscript. 


\section{References}

1. Fierson WM, American Academy of Pediatrics Section on O, American Academy of O, American Association for Pediatric O, Strabismus, American Association of Certified O. Screening examination of premature infants for retinopathy of prematurity. Pediatrics. Jan 2013;131(1):189-195.

2. World Health Organization. WHO | Vision impairment and blindness. Geneva:WHO. [Updated October 2017]; (Accessed 25 January 2018, http://www.who.int/mediacentre/factsheets/fs282/en/. Accessed 25 January 2018).

3. Furtado JM, Lansingh VC, Carter MJ, et al. Causes of blindness and visual impairment in Latin America. Surv Ophthalmol. Mar-Apr 2012;57(2):149-177.

4. Chan-Ling T, Gock B, Stone J. The effect of oxygen on vasoformative cell division. Evidence that 'physiological hypoxia' is the stimulus for normal retinal vasculogenesis. Invest Ophthalmol Vis Sci. Jun 1995;36(7):1201-1214.

5. Raghuveer TS, Bloom BT. A paradigm shift in the prevention of retinopathy of prematurity. Neonatology. 2011;100(2):116-129.

6. Smith LE. Pathogenesis of retinopathy of prematurity. Growth Horm IGF Res. Jun 2004;14 Suppl A:S140-144.

7. Saugstad OD, Aune D. Optimal oxygenation of extremely low birth weight infants: a meta-analysis and systematic review of the oxygen saturation target studies. Neonatology. 2014;105(1):55-63.

8. Gilbert C, Foster A. Childhood blindness in the context of VISION 2020--the right to sight. Bull World Health Organ. 2001;79(3):227-232. 
9. Heidary G, Vanderveen D, Smith LE. Retinopathy of prematurity: current concepts in molecular pathogenesis. Semin Ophthalmol. Mar-Apr 2009;24(2):77-81.

10. Beharry KD, Valencia GB, Lazzaro DR, Aranda JV. Pharmacologic interventions for the prevention and treatment of retinopathy of prematurity. Semin Perinatol. Apr 2016;40(3):189-202.

11. Lapillonne A, Moltu SJ. Long-Chain Polyunsaturated Fatty Acids and Clinical Outcomes of Preterm Infants. Ann Nutr Metab. 2016;69 Suppl 1:35-44.

12. Skouroliakou M, Konstantinou D, Koutri K, et al. A double-blind, randomized clinical trial of the effect of omega-3 fatty acids on the oxidative stress of preterm neonates fed through parenteral nutrition. Eur J Clin Nutr. Sep 2010;64(9):940-947.

13. Connor KM, SanGiovanni JP, Lofqvist C, et al. Increased dietary intake of omega-3polyunsaturated fatty acids reduces pathological retinal angiogenesis. Nat Med. Jul 2007;13(7):868-873.

14. Vayalthrikkovil S, Bashir RA, Rabi Y, et al. Parenteral Fish-Oil Lipid Emulsions in the Prevention of Severe Retinopathy of Prematurity: A Systematic Review and Meta-Analysis. Am J Perinatol. Jun 2017;34(7):705-715.

15. Fang JL, Sorita A, Carey WA, Colby CE, Murad MH, Alahdab F. Interventions To Prevent Retinopathy of Prematurity: A Meta-analysis. Pediatrics. Apr 2016;137(4).

16. Lien EL. Toxicology and safety of DHA. Prostaglandins Leukot Essent Fatty Acids. Aug-Sep 2009;81(2-3):125-132.

17. WMA General Assembly [World Medical Association]. Ethical Principles for Medical Research Involving Human Subjects. Seoul, Korea. [Updated October 2008] (Accessed 20/06/2018, at https://www.wma.net/wp-content/uploads/2016/11/DoHOct2008.pdf) 
18. Saghaei M. Random allocation software for parallel group randomized trials. $B M C$ Med Res Methodol. Nov 9 2004;4:26.

19. Health Ministery. Guidelines of Clinical Practice. Detection, Diagnosis and Treatment of Retinopathy of Prematurity in the second and third level of attention. [Spanish; Secretaría de Salud. Guia de Practica Clinica. Detección, Diagnóstico y Tratamiento de Retinopatía del Prematuro en el Segundo y Tercer Nivel de Atención]. Mexico City: [Updated 2nd July 2015, cited 2010], (Accessed 20/06/2018 at http://cenetec-difusion.com/gpc-sns/?p=909).

20. Section on Ophthalmology American Academy of P, American Academy of O, American Association for Pediatric O, Strabismus. Screening examination of premature infants for retinopathy of prematurity. Pediatrics. Feb 2006;117(2):572576.

21. International Committee for the Classification of Retinopathy of P. The International Classification of Retinopathy of Prematurity revisited. Arch Ophthalmol. Jul 2005;123(7):991-999.

22. Jones JG, MacKinnon B, Good WV, et al. The early treatment for ROP (ETROP) randomized trial: study results and nursing care adaptations. Insight. Apr-Jun $2005 ; 30(2): 7-13$.

23. Repka MX, Tung B, Good WV, et al. Outcome of eyes developing retinal detachment during the Early Treatment for Retinopathy of Prematurity Study (ETROP). Arch Ophthalmol. Jan 2006;124(1):24-30.

24. Network TIN. The CRIB (clinical risk index for babies) score: a tool for assessing initial neonatal risk and comparing performance of neonatal intensive care units. The International Neonatal Network. Lancet. Jul 24 1993;342(8865):193-198. 
25. Wyckoff MH, Aziz K, Escobedo MB, et al. Part 13: Neonatal Resuscitation: 2015 American Heart Association Guidelines Update for Cardiopulmonary Resuscitation and Emergency Cardiovascular Care (Reprint). Pediatrics. Nov 2015;136 Suppl 2:S196-218.

26. Sarkadi-Nagy E, Wijendran V, Diau GY, et al. Formula feeding potentiates docosahexaenoic and arachidonic acid biosynthesis in term and preterm baboon neonates. J Lipid Res. Jan 2004;45(1):71-80.

27. Leaf AA, Leighfield MJ, Costeloe KL, Crawford MA. Factors affecting long-chain polyunsaturated fatty acid composition of plasma choline phosphoglycerides in preterm infants. J Pediatr Gastroenterol Nutr. Apr 1992;14(3):300-308.

28. Bernabe-Garcia M, Lopez-Alarcon M, Salgado-Sosa A, et al. Enteral Docosahexaenoic Acid Reduces Analgesic Administration in Neonates Undergoing Cardiovascular Surgery. Annals of Nutrition and Metabolism. 2016 2016;69(2):150160.

29. Bernabe-Garcia M, Lopez-Alarcon M, Villegas-Silva R, et al. Beneficial Effects of Enteral Docosahexaenoic Acid on the Markers of Inflammation and Clinical Outcomes of Neonates Undergoing Cardiovascular Surgery: An Intervention Study. Annals of Nutrition and Metabolism. 2016 2016;69(1):15-23.

30. Evershed R. Gas chromatography of lipids. In: Hamilton RJ HS, ed. Lipid analysis. A practical approach. Oxford: Oxford University Press; 1992:113-151.

31. Beken S, Dilli D, Fettah ND, Kabatas EU, Zenciroglu A, Okumus N. The influence of fish-oil lipid emulsions on retinopathy of prematurity in very low birth weight infants: a randomized controlled trial. Early Hum Dev. Jan 2014;90(1):27-31. 
32. Lopez-Alarcon M, Bernabe-Garcia M, del Valle O, Gonzalez-Moreno G, MartinezBasilea A, Villegas R. Oral administration of docosahexaenoic acid attenuates interleukin-1beta response and clinical course of septic neonates. Nutrition. Apr 2012;28(4):384-390.

33. Lopez-Alarcon M, Bernabe-Garcia M, Del Prado M, et al. Docosahexaenoic acid administered in the acute phase protects the nutritional status of septic neonates. Nutrition. Jul-Aug 2006;22(7-8):731-737.

34. Cayabyab R, Ramanathan R. Retinopathy of Prematurity: Therapeutic Strategies Based on Pathophysiology. Neonatology. 2016;109(4):369-376.

35. Gong Y, Fu Z, Liegl R, Chen J, Hellstrom A, Smith LE. omega-3 and omega-6 longchain PUFAs and their enzymatic metabolites in neovascular eye diseases. Am J Clin Nutr. Jul 2017;106(1):16-26.

36. Stahl A, Krohne TU, Sapieha P, et al. Lipid metabolites in the pathogenesis and treatment of neovascular eye disease. Br J Ophthalmol. Nov 2011;95(11):1496-1501.

37. Sennlaub F, Valamanesh F, Vazquez-Tello A, et al. Cyclooxygenase-2 in human and experimental ischemic proliferative retinopathy. Circulation. Jul 15 2003;108(2):198204.

38. Beauchamp MH, Martinez-Bermudez AK, Gobeil F, Jr., et al. Role of thromboxane in retinal microvascular degeneration in oxygen-induced retinopathy. J Appl Physiol (1985). Jun 2001;90(6):2279-2288.

39. Settimio R, Clara DF, Franca F, Francesca S, Michele D. Resolvin D1 reduces the immunoinflammatory response of the rat eye following uveitis. Mediators Inflamm. 2012;2012:318621. 
40. Sapieha P, Stahl A, Chen J, et al. 5-Lipoxygenase metabolite 4-HDHA is a mediator of the antiangiogenic effect of omega-3 polyunsaturated fatty acids. Sci Transl Med. Feb 9 2011;3(69):69ra12.

41. Szymczak M, Murray M, Petrovic N. Modulation of angiogenesis by omega-3 polyunsaturated fatty acids is mediated by cyclooxygenases. Blood. Apr 1 2008;111(7):3514-3521.

42. Huun MU, Garberg HT, Escobar J, et al. DHA reduces oxidative stress following hypoxia-ischemia in newborn piglets: a study of lipid peroxidation products in urine and plasma. J Perinat Med. Feb 23 2018;46(2):209-217.

43. Pawlik D, Lauterbach R, Walczak M, Hurkala J, Sherman MP. Fish-oil fat emulsion supplementation reduces the risk of retinopathy in very low birth weight infants: a prospective, randomized study. JPEN J Parenter Enteral Nutr. Aug 2014;38(6):711716.

44. D'Ascenzo R, Savini S, Biagetti C, et al. Higher docosahexaenoic acid, lower arachidonic acid and reduced lipid tolerance with high doses of a lipid emulsion containing 15\% fish oil: a randomized clinical trial. Clin Nutr. Dec 2014;33(6):10021009.

45. Vlaardingerbroek H, Vermeulen MJ, Carnielli VP, Vaz FM, van den Akker CH, van Goudoever JB. Growth and fatty acid profiles of VLBW infants receiving a multicomponent lipid emulsion from birth. J Pediatr Gastroenterol Nutr. Apr $2014 ; 58(4): 417-427$.

46. Najm S, Lofqvist C, Hellgren G, et al. Effects of a lipid emulsion containing fish oil on polyunsaturated fatty acid profiles, growth and morbidities in extremely premature infants: A randomized controlled trial. Clin Nutr ESPEN. Aug 2017;20:17-23. 
47. Di Fiore JM, Bloom JN, Orge F, et al. A higher incidence of intermittent hypoxemic episodes is associated with severe retinopathy of prematurity. J Pediatr. Jul 2010;157(1):69-73.

48. Fairchild K, Mohr M, Paget-Brown A, et al. Clinical associations of immature breathing in preterm infants: part 1-central apnea. Pediatr Res. Jul 2016;80(1):21-27.

49. Lofqvist CA, Najm S, Hellgren G, et al. Association of Retinopathy of Prematurity With Low Levels of Arachidonic Acid: A Secondary Analysis of a Randomized Clinical Trial. JAMA Ophthalmol. Mar 1 2018;136(3):271-277.

50. Calkins KL, Venick RS, Devaskar SU. Complications associated with parenteral nutrition in the neonate. Clin Perinatol. Jun 2014;41(2):331-345.

\section{Figure}

Figure 1. Flow of infants through the study. DHA, Docosahexaenioc acid; ITT, Intention to treat; PDA, Patent Ductus Arteriosus; ROP, Retinopathy of Prematurity. 